\title{
Desarrollo institucional y legal de la Seguridad Social en Argentina
}

Institutional and legal development of Social

Security in Argentina

Desenvolvimento institucional e jurídico

da Previdência Social na Argentina

Développement institutionnel et juridique

de la sécurité sociale en Argentine

阿根廷社会保障的制度和法律发展

\begin{tabular}{l|l} 
Diego Gabriel Presa & $\mid \begin{array}{l}\text { Universidad Nacional de La Plata, } \\
\text { Argentina }\end{array}$
\end{tabular}

Revista Derechos en Acción ISSN 2525-1678/ e-ISSN 2525-1686

Año 4/Nº 13, Primavera 2019 (21 septiembre a 20 diciembre), 345-357

DOl: https://doi.org/10.24215/25251678e341

ORCID: https://orcid.org/0000-0003-3315-9301

Recibido: 07/06/2019

Aprobado: 25/09/2019

Resumen: El presente se corresponde no solo con la historia y evolución del derecho previsional en la República Argentina, sino también con su historia institucional legal, desde comienzo de nuestra historia como Nación hasta nuestros días, llegando a la ley 24241 y sus modificatorias que han permitido incluir bajo planes de moratorias previsionales, un porcentaje cercano al $97 \%$ de la población de adultos mayores.

Palabras claves: ley 24241 - ley 24463 - solidaridad previsional - jubilaciones - ANSeS - moratorias - ley 27260 - reparación histórica - adultos - mayores

1 Abogado en derecho previsional, profesor adjunto de Historia Constitucional de FCJ y $S$ de UNLP, asesor de la Honorable Cámara de Diputados de la Provincia de Buenos Aires. dgpresa@yahoo.com.ar 
Abstract: The present corresponds not only with the history and evolution of pension law in the Argentine Republic, but also with its legal institutional history, from the beginning of our history as a Nation to our days, arriving at Law 24241 and its amendments that have allowed to include under pension moratorium plans, a percentage close to $97 \%$ of the population of older adults.

Keywords: law 24241 - law 24463 - social security solidarity - pensions - ANSeS - moratoriums - law 27260 - historical repair - adults - seniors

Resumo: 0 presente corresponde não somente com a história e evolução do direito previdenciário na República Argentina, senão também com sua história institucional legal, desde o início de nossa história como nação até nossos dias, alcançando a Lei 24241 e suas emendas que nos permitiram incluir sob planos de moratória previdenciárias, um percentual próximo a $97 \%$ da população idosa.

Palavras-chave: lei 24241 - lei 24463 - solidariedade previdenciária aposentadoria - ANSeS - moratórias - lei 27260 - reparação histórica - adultos - idosos

Résumé: Ce travail n'est pas seulement le récit historique de l'évolution du droit des pensions en République d'Argentine, mais aussi celui de son histoire institutionnelle juridique, depuis le début de notre histoire en tant que nation jusqu'à nos jours, jusqu'à la loi 24241 et ses modifications qui nous ont permis d'inclure dans le cadre de plans de moratoire sur les pensions, un pourcentage proche de $97 \%$ de la population de personnes agées.

Mot-clés: Ioi 24241 - loi 24463 - pension solidaire - retraite - ANSeS moratoires - loi 27260 - réparation historique - adultes - seniors

摘要：目前, 这不仅与阿根廷共和国的退休金法的历史和演变有关, 而且还与从我们作为国家的历史到今天的第24241号法律及其修正 案相关的法律制度的历史。允许将其纳入养老金计划, 这一比例接 近老年人口的 $97 \%$ 。

关键字：24241号法律-24463号法律-退休金团结-退休--暂停-27260 号法律--成人-老年人 


\section{Introducción}

Este trabajo no es solo un relato histórico del derecho previsional en la República Argentina, sino de su desarrollo institucional y legal.

El lector podrá conocer los antecedentes previsionales que datan de 1810 hasta la actualidad, llegando a la complejidad jurídico actual, que incluye regímenes diferenciales en edad y aportes, así como moratorias o planes de inclusión previsionales, que han permitido llegar a tener bajo protección del sistema de seguridad social a la gran mayoría de la población adulta mayor.

\section{Desarrollo institucional (1810-1967)}

La seguridad social en Argentina, la podemos remontar a 1810 , pero basados en un sistema graciable en su totalidad y que no se nutría por contribuciones de ninguna índole.

Estos beneficios graciables amparaban necesidades nacidas de las guerras de la independencia fundamentalmente.

En 1858 con la instalación del Hotel de Inválidos, que con el tiempo se transformó en Asilo de Ancianos, hay una ayuda del Estado hacia mendigos o inválidos, hotel que estuvo en funcionamiento hasta 1978.

En 1877 fue promulgada la ley $877{ }^{2}$ que estableció jubilaciones vitalicias y no contributivas para Ministros de la Corte Suprema de la Nación y Jueces Nacionales que, con el requisito de 10 años en el ejercicio del cargo, accedían al goce íntegro de su sueldo en forma vitalicia, y no se transmitía a la viuda.

La ley $1420^{3}$ de 1884 conocida como de educación común, otorgaba a preceptores y subpreceptores una pensión por enfermedad y una pensión por retiro, asimismo fijaba una

2 Promulgada el 30-09-1877, presidencia de Nicolás Avellaneda 1874-1880

3 Promulgada el 26-06-1884, presidencia de Julia A Roca 1880-1886 
contribución del 2 por ciento del sueldo mensual. Para esto debía contar con 10 años de servicios. A través de la ley $1909^{4}$ de 1886 son comprendidos en el régimen jubilatorio los maestros también.

En el año 1904, con la promulgación de la Ley 4.3495, que crea la Caja Nacional de Jubilaciones y Pensiones para Funcionarios, Empleados, y Agentes Civiles de la Administración, se da inicio a la etapa orgánica de los regímenes previsionales en la República Argentina. Esta ley fue sancionada el día 20 de septiembre de 1904, es por ello que en ese día se celebra el día del jubilado.

Fue una de las principales leyes que estableció los principios que se transmitieron en las leyes que vendrían con el tiempo, como por ejemplo la contribución forzosa al régimen, que los fondos eran de los aportantes, o la retención de la mitad del primer mes de sueldo del empleado ingresado en la administración, nace la solidaridad del sistema entre pares.

Al promulgarse esta ley, surgieron cajas de carácter gremial o por sectores de la sociedad como por ejemplo:

En 1915 Caja para el personal ferroviario ley $9653^{6}$, el defecto de esta ley era la restricción que pesaba sobre el derecho de huelga, donde cualquier trabajador que plegara a un paro sindical, si era despedido perdía los aportes realizados y el acceso a jubilarse.

En 1921 Caja para el personal de Servicios Públicos, ley $11.110^{7}$, que comprendía a personal de empresas de tranviarios, telefónicos, telégrafos, gas y electricidad.

4 Publicada el 24-11-1886 presidencia de Miguel Juárez Celman 1886-1890 renuncio por la revolución del parque

5 Publicada el 27-09-1904, Segunda presidencia de Julio Argentino Roca 1898-1904

6 Publicada el 05-07-1915, presidencia de Victorino de la Plaza 1914-1916, reemplazo a Roque Sáenz Peña quien falleció en el cargo

7 Publicada el 24-02-1921, presidencia de Hipólito Yrigoyen 1916-1922 
En 1923 Caja para el personal bancario y de compañías de seguros, ley $11232^{8}$.

En 1939 Caja para el personal del periodismo y actividades gráficas, ley $12581^{9}$.

En 1944, Caja para el personal de comercio y actividades civiles, decreto ley $31665^{10}$.

En 1946, Caja para el personal de la industria privada, decreto ley $13937^{11}$.

En 1949, con la reforma constitucional, se introdujo por vez primera la seguridad social. Aparecen contemplados los derechos del trabajador en su artículo 37 con un inciso especial sobre el tema como era el séptimo "...Derecho a la seguridad social: El derecho de los individuos a ser amparados en los casos de disminución, suspensión o pérdida de su capacidad para el trabajo, promueve la obligación de la sociedad de tomar unilateralmente a su cargo las prestaciones correspondientes o de promover regímenes de ayuda mutua obligatoria destinados unos y otros, a cubrir o complementar las insuficiencias o inaptitudes propias de ciertos períodos de la vida o las que resulten de infortunios provenientes de riesgos eventuales..." promoviendo la ayuda mutua obligatoria, resultando ser la jubilación, ya sea la común u ordinaria, por incapacidad, por edad avanzada o vejez, o por fallecimiento en actividad, una pensión para la familia que queda desamparada.

Esta regulación de la ley $14370^{12}$ introduce al Estado al cuidado de su población en general, a cubrir ese desamparo que con el tiempo nos afecta a todos y que causa incertidumbre, pero sin embargo ha recibido el nombre de jubilación que

\footnotetext{
8 Publicada el 19-11-1923, presidencia de Marcelo T de Alvear 1922-1928

9 Publicada el 06-07-1939, presidencia de Roberto M Ortiz, 1938-1942, falleció en el cargo

10 Publicada el 06-12-1944,presidencia de facto de Edelmiro J Farrel 1944-1946

11 Publicada el 07-11-1950, presidencia de Juan Domingo Perón 1946-1952

12 Publicada el 18-10-1954, segunda presidencia de Juan Domingo Perón 1952-1955 interrumpida por un golpe cívico-militar
} 
deriva de júbilo, de alegría, donde justamente el más anciano, por su esfuerzo, por su trabajo, por su aporte a la sociedad y al desarrollo de la misma, recibe un premio para tener un descanso y vida adecuada.

En 1954, se dictó la ley 14370, se lleva a la unificación de los sistemas y se pasa al sistema de reparto y la ley $14397^{13}$ de trabajadores autónomos.-

Es a partir de 1954, con la sanción de la Ley 14.370, que se implementa un cambio de paradigma, pasando de un sistema individualista a un sistema solidario, por el cual se establece un pacto intergeneracional donde las generaciones más jóvenes solventaran con aportes a las generaciones más viejas o en su caso por invalidez o fallecimiento

En 1955, se creó la Caja de trabajadores rurales.

En el gobierno de facto de la dictadura cívico-militar de 1955 a 1958, se establece el régimen previsional para el servicio doméstico, a través del Decreto Ley $11.911 / 56^{14}$, de alcance nacional, el capital de la caja se conformaría con el aporte obligatorio del $5 \%$ del sueldo por parte del afiliado y con la contribución patronal del $7 \%$.

En 1957, se introdujo en la Constitución Nacional el artículo 14 bis que reflejo postulados del constitucionalismo social, articulo que nos rige hoy en día en la materia y que establece el precepto de "Jubilación y Pensiones móviles", el cual la Corte Suprema de Justicia de la Nación y fundamentalmente a partir del año 2005 ha venido esgrimiendo como un precepto que debe ser respetado con fallos como Sánchez María del Carmen ${ }^{15}$, Badaro Adolfo Valentín ${ }^{16}$, y el ultimo Blanco Lucio

\footnotetext{
13 Publicada el 21-01-1955, segunda presidencia de Juan Domingo Perón

14 Publicada el 16-07-1956, presidencia de facto de Pedro Aramburu, 1955-1958 integro un gobierno cívico militar

15 CSJN colección fallos 328:2833 de fecha 17-05-2005

16 CSJN colección fallos 330:4866 de fecha 26-11-2007
} 
Orlando $^{17}$, todos contra el ANSeS (Administración Nacional de Seguridad Social).

En 1958, se introdujo el 82\% móvil de la última remuneración asignada al cargo, oficio o funcione que detento el titular, según el artículo 28 de la ley $14499^{18}$.

Esta ley se aplicó a las Cajas de Previsión del Personal del Estado, Personal Ferroviario, Servicios Públicos, Bancarios y de Seguros, Periodismo, Navegación, Comercio y Actividades Civiles, Industria y Trabajadores Rurales, entre otras.

Creó un Fondo Compensador de Inversiones y Acumulación, a cargo del Instituto Nacional de Previsión Social, cuyos recursos se formaban con los excedentes de las Cajas Nacionales que lo integraban. El Fondo seria usado para:

a) Subsanar los déficits existentes o que surgieran;

b) Financiar inversiones en producción de energía, combustibles y siderurgia;

c) Mejorar y ampliar los sistemas de transportes y vialidad

El legislador no pudo prever en la ley, el aumento del número de jubilados y pensionados en general, la pérdida del poder adquisitivo por un problema que asomaba que era la inflación y la inexistencia de inversiones que protegieran los fondos, llevando al desfinanciamiento y quiebra del sistema.

En 1967 y ya en la dictadura cívico militar de 1966 a 1973, se dicta la Ley $15.575^{19}$, provoca una transformación en el sistema reduciendo de trece a tres el número de cajas en funcionamiento, quedando así las Cajas de:

\footnotetext{
17 CSJN colección fallos 341:1924 de fecha 18-12-2018

18 Publicada el 17-10-1958, presidencia de Arturo Frondizi, 1958-1962 mandato interrumpido por un golpe cívico militar.

19 Publicada el 24-11-1960, presidencia de Arturo Frondizi, 1958-1962 mandato interrumpido por un golpe cívico militar
} 


\section{Industria, Comercio y Actividades Civiles. Estado y Servicios Públicos. Autónomos}

En el año 1969, se procede a la fusión de las dos primeras cajas mencionadas anteriormente, conformándose así el régimen de trabajadores en relación de dependencia, bajo la normativa de la Ley $18.037^{20}$, mientras que con la tercera se define el régimen de trabajadores autónomos o independientes, normados con la Ley $18.038^{21}$.

Estas dos leyes estandarizaron los requisitos jubilatorios y los mecanismos de financiamiento, se elevó la edad jubilatoria, se elevó el número de años de aporte, y se determinó que los haberes jubilatorios estarían asociados con la historia individual laboral de cada trabajador, tomándose los últimos diez años de aportes pero determinando el haber jubilatorio con los mejores tres años aportados en esos últimos diez.

La dictadura cívico-militar de 1976 a 1983, se dio un nuevo texto ordenado a las leyes 18037 y 18038 en 1980, se impulsó una reforma de carácter regresiva del sistema previsional, al solo efecto de sostener su financiamiento sobre recursos extraídos a las clases sociales medias y bajas.

Esto implicó en los hechos una transferencia de recursos, eliminando parte de las contribuciones patronales, reemplazándolas por la ampliación del Impuesto al Valor Agregado (IVA) ${ }^{22}$, impuesto que es pagado por todos diríamos que todo el tiempo, siendo el trabajador y el pueblo en general un socio en las perdidas.

Asimismo, el Estado para sostener el sistema restringió la movilidad en su aplicación utilizándola como variable de ajuste, dando comienzo a los denominados juicios de reajuste por

\footnotetext{
20 Publicada el 10-01-1969, presidencia de facto de Juan Carlos Ongania, 1966-1970

21 Publicada el 10-01-1969, presidencia de facto de Juan Carlos Ongania, 1966-1970

22 Ley 20631, publicada 31-12-1973, tercera presidencia de Juan Domingo Perón 1973-1974 falleció en el cargo
} 
parte de los jubilados, juicios que consisten en un daño en el haber previsional que se reclama ante la justicia y es esta la que fija la movilidad y su pauta de actualización.

En 1986, el presidente Raúl Alfonsín ${ }^{23}$ decretó la Emergencia Previsional, llevando al congelamiento de las demandas y la ejecución de sentencias en contra del Estado.

En 1990, la ley $23769^{24}$, creo el Instituto Nacional de Previsión Social.

En 1991 y por decreto $2284^{25}$, vulgarmente conocido como de desregulación económica, se creó el Sistema Único de Seguridad Social, dependiente del Ministerio de Trabajo y Seguridad Social y por decreto $2741 / 91^{26}$, se crea ANSeS, Administración Nacional de Seguridad Social.

Estas dos leyes con sus modificaciones y textos ordenados llegaron vigentes hasta julio de 1994, con la sanción de la Ley $24.241^{27}$, donde se creó el un nuevo sistema denominado de capitalización que conviviría con el viejo sistema solidario.

A partir de esta ley y de la firma de pactos fiscales varias provincias han transferido sus regímenes previsionales públicos a la Nación, entre ellas Salta, Jujuy, La Rioja, Tucumán, Mendoza, San Juan, San Luis y Entre Ríos.

Entre las leyes emblemáticas que han dispuesto modificaciones o complementado el sistema de la ley 24241, se encuentra la ley $24463^{28}$ denominada de solidaridad previsional, la cual nunca cumplió su objetivo de instalar la movilidad de haberes que requería el sistema, ley que ha sido y es impugnada actualmente por distintos planteos judiciales.

\footnotetext{
23 Raúl Ricardo Alfonsín, primer presidente del retorno a la democracia 1983-1989

24 Publicada el 19-01-1990, primera presidencia de Carlos Saúl Menem, 1989-1995

25 Publicada el 01-11-1991, Decreto de Necesidad y Urgencia, presidencia de Carlos Saúl Menem 1989-1995

26 Publicado el 08-01-1992, Decreto presidencia de Carlos Saúl Menem 1989-1995

27 Publicada el 18-10-1993, presidencia de Carlos Saúl Menem 1989-1995

28 Publicada el 30-03-1995, presidencia de Carlos Saúl Menem 1989-1995
} 
La ley 24241, estableció el sistema de reparto público y la capitalización individual, el primero, administrado por el Estado y donde el trabajador debía optar por permanecer en él dentro de los 30 días de su primer empleo, caso contrario sería derivado a la AFJP que cobrara menor comisión en el momento del traspaso, no pudiendo retornar más al sistema público, esto garantizaría al sistema privado clientes constantes que llegarían por no ejercer la opción.

El otro subsistema era el de las AFJP o Administradoras de Fondos de Jubilaciones y Pensiones (AFJP) y Compañías de Seguro de Retiro (CSR), claramente del sector privado, modelo copia del Chile de la de década de los años 80, otorgando iguales beneficios al sistema público, pero con una cuenta individual de cada trabajador que cobraría según su expectativa de vida a partir de la edad de 65 años para los hombres y 60 años para las mujeres.

El sistema era de carácter individualista, renunciando al principio de solidaridad fundamentalmente, donde el trabajador que no concretara una cantidad de aportes razonables -digamos los 30 años- no accedía a un derecho jubilatorio que le permitiera vivir dignamente. A esto se sumó la crisis económica de 2001 y 2002 donde el desempleo llevara a los trabajadores al no aporte y a no acceder a las prestaciones.

Otras de las leyes que modifico el sistema fue la ley $25994^{29}$ de 2005, que posibilito la entrada de personas que no podían acceder a las prestaciones previsionales, ha poder pagar moratorias, que implicaron la obtención del beneficio, además por el artículo $2^{\circ}$ de dicha ley se posibilito obtener un beneficio con menores requisitos para las personas que habían "sufrido" el proceso de privatización de los años 90, los menores requisitos era poder completar los 30 años de aportes sino los tenia, es decir la persona que había sido cesanteada con las privatizaciones de los años 90, podría sumar a sus años de aportes los

29 Publicada el 07-01-2005, presidencia de Néstor Carlos Kirchner 2003-2007 
años de moratoria necesarios, y la edad sería de 60 años para hombres y 55 para mujeres, esta ley tuvo una vigencia temporal.

Esta ley 25994, fue complementada por una ley anterior que se dispuso su vigencia nuevamente que fue la ley $24476^{30}$ y que por un decreto reglamentario 1454/2005, restableció una nueva moratoria con alcances de tiempo e ingresos, hasta el mes de septiembre de 1993, es decir aquí las personas podrían comprar años en moratoria hasta el mensual 09/1993, lo que llevaba a su cierre con el paso del tiempo.

La ley $26222^{31}$ de 2007, estableció la libre elección el sistema a adoptar, es decir reparto o capitalización, recordemos que era una opción que no existía debido que si el titular no manifestaba quedarse en el régimen publico dentro de los 30 días de su primer empleo pasaba directamente a capitalización, asegurando clientes cautivos a las ex AFJP.

La ley $26417^{32}$ del año 2008, instrumento la movilidad de las prestaciones previsionales con dos aumentos por años establecidos para los meses de marzo y septiembre de cada año.

En el año 2008 y por ley $26425^{33}$, se unifico el sistema público y privado en un solo régimen público y de reparto, los motivos de esta unificación y el cierre de las AFJP, era porque la mayoría de los trabajadores que se jubilaban no tenían más años en el sistema de reparto -anterior a 07/1994- que los años que habían aportado hasta esa fecha, que no eran más de 14 años, desde 1994 a 2008, que pasaba aquí, que los aportes en la cuenta individual, no alcanzaban para cubrir la expectativa de vida, y el aporte en la caja administrada por el estado, paga lo que se denominó el componente público, logrando acceder así siempre a un beneficio mínimo sin ninguna de las mejoras que las AFJP en una especie de canto de sirenas, habían prometido.

\footnotetext{
30 Publicada el 23-11-1995, segunda presidencia de Carlos Saúl Menem 1995-1999

31 Publicada el 26-12-2013, segunda presidencia de Cristina Fernández de Kirchner 2011-2015

32 Publicada el 16-10-2008, primera presidencia de Cristina Fernández de Kirchner 2007-2011

33 Publicada el 09-12-2008, primera presidencia de Cristina Fernández de Kirchner 2007-2011
} 
En diciembre de 2011 fue aprobada la ley $26727^{34}$, que estableció nuevos requisitos para los trabajadores del sector rural o agrario, clasificando al trabajo como insalubre o desgastante.

En septiembre de 2014 se dictó la ley $26970^{35}$, llamada de inclusión previsional, permitió una nueva moratoria y la inclusión de personas al sistema previsional, con una cuota que fue actualizable de acuerdo a los aumentos de las jubilaciones, y donde se procedió a realizar un análisis socio-económico de la persona que pedía acceder al beneficio, dándole la posibilidad a los que no tenían ningún tipo de ingreso, de tener una prestación previsional con obra social.

En el año 2016 se dictó la ley $27260^{36}$ denominada como de reparación histórica, que permite acceder a un arreglo judicial con ANSeS evitando el juicio y obviamente como todo acuerdo ambas partes renuncian a derechos evitando futuros reclamos.

La ley estableció una tercer moratoria previsional, que duro unos meses para los hombres, debido que el hombre que cumplía 65 años, se procedió por parte del organismo a aplicarle la denominada PUAM, Prestación Universal al Adulto Mayor, cerrándole el camino a la moratoria y una prestación más completa como la jubilación, dado que la PUAM no se transmite en formato de pensión.

Más allá de eso, el objetivo central de la ley, se suponía era terminar con los juicios contra el organismo, es decir el denominado juicio de reajuste, en la práctica, el incumplimiento de ANSeS en el pago de lo prometido, el pagar menos aplicando el índice RIPTE, motivó una mayor conflictividad, duplicando el número de sentencia al pago desde la sanción de la ley.

Ha generado también en los tribunales federales y de seguridad social una mayor cantidad de ejecuciones contra el Estado; se establecieron secretarías completas dedicas a esta segunda etapa.

\footnotetext{
34 Publicada el 28-12-2011, segunda presidencia de Cristina Fernández de Kirchner 2011-2015

35 Publicada el 10-09-2014, segunda presidencia de Cristina Fernández de Kirchner 2011-2015

36 Publicada el 22-07-2016, presidencia de Mauricio Macri, 2015-2019
} 


\section{Síntesis final}

La historia escrita del sistema previsional argentino, sin dudas unos de los más completos y que mayor cobertura otorga a nivel de Latinoamérica, ha sido como la del país en general, periodos de crecimiento con un sistema estable, otros de crisis profundas, de toma de malas decisiones que llevaron a la quiebra del régimen público y de reparto, pero lo que debemos tener siempre presente, es no volver a caer en el canto de sirena del régimen individual, donde se supone que uno se salva solo y sin ayuda de nadie, debemos tratar de mantener el sistema de solidaridad, ese pacto intergeneracional del que tanto hablamos y que servirá para otorgar futuras prestaciones a los actuales trabajadores, donde el sistema es salvado por todos, comprendiendo a las amas de casa -cuando pagan IVA- a los trabajadores -cuando aportan- a los empleadores -con sus contribuciones- siempre es mejor pagar un poco más y tener un sistema rentable, a tener el conflicto permanente por mal pago.

\section{Bibliografía}

"Derecho a la vejez" fundamentos y alcances, autora María Isolina Dabove, editorial Astrea año 2018.

"Jubilaciones \& Pensiones" 4ta edición, autora María Delia Lodi-Fe, año 2018

"Movilidad Jubilatoria", Informe de la Corte Suprema de Justicia de la Nación Argentina, Secretaria de Jurisprudencia año 2009

"Manual de la Seguridad Social", $2^{\text {a }}$ edición, actualizada y complementada, editorial Abaco de Rodolfo Depalma, Autores Pedro Taddei, Carlos Mongiardino y Reinaldo Naccarato, edición marzo de 2007

Página web www.ingoleg.gob.ar 